\title{
Elaboración de un glosario de términos jurídicos en la asignatura Fundamentos jurídicos y sociales para la ordenación territorial y medioambiental, grado de Geografía y Ordenación del Territorio ${ }^{1}$.
}

\author{
María del Mar Villagrasa $\operatorname{Rozas}^{2}$ y María del Carmen de Guerrero Manso ${ }^{3}$
}

\begin{abstract}
The Legal and Social Fundamentals course for territorial and environmental planning requires the knowledge and use of legal concepts whose terminology is often complex and completely unknown to the average profile of the students of the Degree in Geography and Territorial Planning. Besides, this subject is studied in the first half of the second year of the Degree, when students are not familiar with the basics that make up their curriculum. The purpose of this project is the development of a glossary of legal terms used in the subject, so that a proper definition of these make easier the apprehension of basic and accurate knowledge, essential to get a good academic results.
\end{abstract}

Keywords: glossary, forum, cooperative learning.

\begin{abstract}
Resumen
La asignatura Fundamentos jurídicos y sociales para la ordenación territorial y medioambiental exige el conocimiento y la utilización de conceptos jurídicos cuya terminología suele resultar compleja y totalmente desconocida para el perfil medio de los alumnos del Grado en Geografía y Ordenación del Territorio. A ello se suma el hecho de que la asignatura se curse en el primer semestre del segundo curso de dicho grado, cuando los estudiantes no están familiarizados con los conceptos básicos que conforman su currículo. La finalidad del proyecto es la elaboración de
\end{abstract}

\footnotetext{
${ }^{1}$ El proyecto que presentamos ha sido aprobado en el marco del Programa de Incentivación de la Innovación Docente de la Universidad de Zaragoza (PIIDUZ_14_67), dentro de la convocatoria de Innovación Docente 2014-2015.

2 Profesora colaboradora de la Universidad de Zaragoza, Departamento de Derecho Público, mvilla@unizar.es

${ }^{3}$ Profesora Ayudante Doctora de la Universidad de Zaragoza, Departamento de Derecho Público, carmeng@unizar.es
} 
Elaboración de un glosario de términos jurídicos en la asignatura Fundamentos jurídicos y sociales para la ordenación territorial y medioambiental, grado de Geografía y Ordenación del Territorio

un glosario de términos jurídicos utilizados en la asignatura, de modo que una adecuada definición de los mismos facilite la aprehensión de conocimientos básicos y precisos, imprescindibles para obtener un buen rendimiento académico.

Palabras clave: glosario, foro, aprendizaje colaborativo

\section{Introducción}

El Espacio Europeo de Educación Superior exige un cambio en las metodologías docentes tradicionales que las nuevas tecnologías pueden ofrecer. La entrada de las TICs en la Universidad ha supuesto un reto para los profesores, al mismo tiempo que una oportunidad de mejora en los métodos pedagógicos y en las posibilidades de aprendizaje de los alumnos.

La plataforma de aprendizaje Moodle dispone de múltiples herramientas que posibilitan el diseño del entorno de enseñanza-aprendizaje. La función principal como profesor de un asignatura del aula virtual consiste en dotar de contenido al curso como escaparate de los materiales que los alumnos necesitan para aprender su materia pero, además, resulta necesario manejar herramientas de comunicación e interacción, y diseñar actividades docentes que ayuden a los estudiantes en su tarea de aprendizaje de forma activa, interaccionando con el profesor y sus compañeros (HOLGADO, 2011). Para ello, Moodle dispone de un módulo colaborativo en el que están los foros, las bases de datos, el glosario y la wiki.

En esta actividad hemos decidido utilizar el glosario y el foro para experimentar el aprendizaje colaborativo, pero hubiera podido hacerse igualmente por medio de una wiki. El aprendizaje tiene lugar durante el proceso de elaboración del glosario y concluye con un recurso didáctico (producto final) que servirá como referencia de consulta y material de estudio. La posibilidad de crear e investigar las definiciones por parte de los estudiantes les facilita la aprehensión de conocimientos básicos y precisos de la asignatura.

El interés por hacer posible la adquisición de un vocabulario técnico-jurídico de manera dinámica y activa nos llevó a idear esta actividad de forma que fuera elaborada por los alumnos con la supervisión de las profesoras y resultara útil para todo el grupo, tanto para los que asistían a clase como para los que no podían asistir por motivos laborales o personales (algunos alumnos cursan el grado en Geografía y Ordenación del Territorio como un segundo grado complementario al de Historia del Arte o Historia).

Por otro lado, Fundamentos jurídicos y sociales para la ordenación territorial y medioambiental, es la única asignatura de contenido jurídico que estudian los alumnos de este Grado. Algunos de los conceptos básicos utilizados en esta asignatura son compartidos con otras materias, pero muchos de ellos son propiamente jurídicos, por lo que los alumnos 
carecen de conocimientos previos y, pese a que lo aprendido en esta asignatura les será de gran utilidad en su ejercicio profesional, en cierta medida constituye un conocimiento exclusivo de Derecho. Así, el alumno se enfrenta a una disciplina nueva con su propio lenguaje técnico que debe dominar para aprobar la asignatura.

Con la finalidad de facilitar el proceso de enseñanza-aprendizaje se ha llegado a la conclusión de que la introducción de los términos técnicos deberá hacerse de forma gradual a lo largo del curso, y por bloques temáticos. Además es preciso destacar que una de las competencias específicas de la Titulación es, precisamente, el dominio de la terminología científica de las diversas ramas de la Geografía y de la Ordenación del Territorio.

\section{Objetivos}

El objetivo fundamental de esta metodología o práctica docente es conseguir un recurso didáctico: un glosario básico de términos jurídicos relacionados con la ordenación del territorio, el urbanismo y el medio ambiente, que sea realizado por los propios alumnos, ayudando de esta manera al profesor a adquirir y completar material didáctico para la enseñanza de la asignatura, y que ayude a los alumnos a comprender y asimilar el contenido de la asignatura. El glosario puede ser utilizado también para las asignaturas de cursos posteriores en las que se utilizarán términos jurídicos como conceptos transversales.

Conjuntamente se pretende fomentar el trabajo en grupo entre los estudiantes. Esta es una de las competencias transversales que se establecen para el Grado, pero en ciertas ocasiones no se prevén espacios adecuados para que los alumnos puedan desarrollar sus habilidades y adquirir esta competencia. En este caso se pretende que trabajen en grupos, de manera colaborativa, para elaborar los diversos términos del glosario.

El tercer objetivo que se pretende lograr es que los alumnos trabajen en la plataforma virtual facilitada por la Universidad de Zaragoza. De esta manera, a través del ADD de Moodle y, más específicamente, de su módulo colaborativo podrán aprender a utilizar diversas herramientas como el foro, las tareas, la subida de archivos, añadir comentarios a un término del glosario... pudiendo posteriormente utilizar este recurso en otras asignaturas.

\section{Desarrollo de la innovación}

El módulo de actividades "Glosario" permite a los participantes crear, y posteriormente acceder, a una lista de definiciones, de estilo similar a la que se contiene en un diccionario. El profesor puede elaborar un glosario con los términos que considera más significativos de la materia que imparte y ponerlo a disposición de los estudiantes para facilitarles la comprensión y el aprendizaje de la materia. Además el texto del glosario puede enriquecerse con imágenes, vídeos, etc, con lo que se puede dotar a los términos de un carácter más visual. Las entradas del glosario pueden agruparse por categorías, si bien en este caso se ha optado por elaborar un glosario general.

Desde la plataforma Moodle puede crearse un glosario principal que será único y editable por el profesor, o uno o varios glosarios secundarios, que pueden ser editados directamente

\section{(c)) EY-NC-ND 2015, Universitat Politècnica de València}

Congreso In-Red (2015) 
Elaboración de un glosario de términos jurídicos en la asignatura Fundamentos jurídicos y sociales para la ordenación territorial y medioambiental, grado de Geografía y Ordenación del Territorio

por los alumnos. Desde el principio se decidió dinamizar el trabajo colaborativo desde el foro para después crear un glosario principal por las profesoras con los términos mejor definidos. De esta manera se ha logrado una gran coordinación entre el trabajo inicial de los alumnos en la elaboración de las definiciones y la posterior selección de ellos por parte de las profesoras.

En cuanto al desarrollo de la experiencia, al tratarse de una asignatura impartida por dos profesoras distintas ha sido necesaria la coordinación y colaboración de ambas. La coordinación tuvo como punto de partida el reparto de los temas del programa que iba a impartir cada una, así como la previsión de un cronograma de actividades docentes para evitar solapamientos y mejorar el proceso de enseñanza.

En la primera fase del proyecto las profesoras elaboraron la lista de voces que iban a formar parte del glosario, en la que figuraban términos de introducción al Derecho, ordenación del territorio, urbanismo y medioambiente. De este modo, los alumnos podrían comenzar el trabajo a partir de los conocimientos y aprendizaje adquiridos en cada clase. También subimos a Moodle una guía con las reglas que debían orientar a los estudiantes en la elaboración de cada término del glosario, mostrando algunos ejemplos, y animamos a los alumnos a asistir a tutorías ante cualquier duda o problema que les pudiera surgir. En total, el glosario estaría formado por 45 voces.

En la segunda fase los estudiantes se distribuyeron en grupos y se les asignaron 8 o 10 voces del glosario para que fueran elaborándolo. El número de términos dependía de la dificultad de los conceptos que debían ser definidos, intentando equiparar la carga de trabajo real exigida a cada grupo. Una vez redactada la propuesta inicial de cada voz, ésta debía enviarse al foro para ser corregida y evaluado por las profesoras, con la participación de los demás alumnos. Se fijó una fecha límite para la entrega, coordinada con el avance del programa de la asignatura.

A medida que los alumnos iban enviando los términos las profesoras procedían a revisarlos, comprobando que estuvieran correctos y fueran comprensibles para un lego en Derecho. Si tras la revisión inicial se consideraba que un término era incorrecto se reenviaba por correo electrónico al miembro del grupo que lo había subido al foro, indicándole que debía rectificarlo y volver a subirlo de nuevo al foro. Si por el contrario, se consideraba adecuada la definición, quedaba guardada en el foro.

Una vez que todos los grupos habían redactado y subido al foro los términos del glosario que se les habían asignado, se les pidió que eligieran los que consideraran más adecuados para ser insertados en el glosario final, puntuando el trabajo de los diferentes grupos en una escala del 1 al 5. Con ello, no sólo se animaba a su participación activa, poniendo en marcha la heteroevaluación, sino que debían ser capaces de hacer una comparación crítica del trabajo de sus compañeros. Sin embargo, los alumnos rechazaron desde el principio esta posibilidad, y muy pocos evaluaron el trabajo de sus compañeros. Pese a ello, todos los grupos eligieron los términos que estaban mejor definidos y resultaban más comprensibles a la hora de preparar el examen final de la asignatura, que se realizaba la última semana de enero, y cuya primera pregunta consiste en la definición de cinco términos del Glosario. 
Al no ser posible la heteroevaluación, en la tercera fase las profesoras prescindieron de ella, y elaboraron el glosario final con los términos mejor definidos por los alumnos.

Por último, en enero de 2015, la semana antes de acabar el cuatrimestre, se pasó a los alumnos un cuestionario para conocer su grado de satisfacción con el desarrollo de la asignatura, las metodologías y recursos docentes utilizados a lo largo del curso y, específicamente, esta nueva experiencia.

La participación en la actividad del glosario formaba parte de la evaluación continua de la asignatura, en la cual los alumnos podían conseguir un 15\% de la nota final realizando además actividades prácticas y utilizando el portafolio de aprendizaje.

El interés de los alumnos por la elaboración y utilización del glosario final se basaba fundamentalmente en que el recurso didáctico obtenido les iba a servir para la preparación de la prueba final a la que se asigna el $60 \%$ de la puntuación de la asignatura, y en la que aparecen preguntas teóricas sobre conceptos jurídicos concretos que aparecen en el temario, y que resultan imprescindibles para comprender el Derecho y, por lo tanto, para poder superar "Fundamentos jurídicos y sociales para la ordenación territorial y medioambiental".

De esta forma se conectaba de manera clara y muy adecuada esta actividad con los resultados de aprendizaje que se definen en la guía docente de la asignatura: conocer y utilizar correctamente la terminología jurídica básica, tanto en la expresión oral como escrita.

Además de la adquisición de conocimientos actualizados del derecho territorial y medioambiental, con esta experiencia los alumnos trabajaron otras competencias transversales que se incluyen en la guía docente, especialmente el desarrollo de aptitudes para seleccionar información e interpretar y valorar de forma crítica la gran cantidad de datos y contenidos que se manejan.

Este tipo de trabajo colaborativo potencia las competencias que son necesarias para el buen aprendizaje en equipo, tales como la capacidad de organizar y planificar, tomar decisiones, habilidades interpersonales, liderazgo, etc. No cabe duda de que el trabajo en equipo que se realiza en modo de e-learning requiere un mayor esfuerzo y compromiso por parte del grupo.

En cuanto a la evaluación de la asignatura, dos de las cinco preguntas del examen final escrito estaban relacionadas con términos del glosario. La primera pregunta consistía en definir cinco conceptos jurídicos básicos, esenciales para entender el Derecho, y que por ese motivo figuraban entre la lista de términos del glosario. La tercera pregunta del examen consistía en exponer de manera abreviada una parte del programa y, para proceder a tal exposición se podían utilizar los términos del glosario.

\section{Resultados}

La participación de los alumnos por grupos fue muy alta y se consiguió el primer objetivo que se pretendía: un recurso didáctico obtenido mediante la colaboración de toda la clase. De los 15 grupos en los que se dividía la clase para hacer trabajos y exposiciones orales participaron 12 en la elaboración del glosario. La queja más común por parte de los

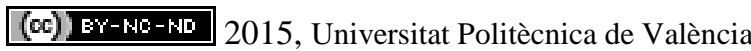


Elaboración de un glosario de términos jurídicos en la asignatura Fundamentos jurídicos y sociales para la ordenación territorial y medioambiental, grado de Geografía y Ordenación del Territorio

alumnos fue que el exceso de trabajo en todas las asignaturas del curso no les permitía invertir tanto tiempo como necesitaban para realizar de manera adecuada su cometido.

Entre los aspectos a mejorar está el hecho de que se observó que la mayor parte del contenido del glosario no se completó con el contenido de las clases, como las profesoras hubieran deseado, sino que la mayoría de los grupos lo realizaron utilizando diversas páginas de internet, fundamentalmente la wikipedia y alguna enciclopedia jurídica, limitándose a “copiar y pegar” y, en algunos casos, sin entender el significado de lo que se aportaba. Por otro lado, algunos grupos enviaron su trabajo al foro en formato pdf, por lo que el resto de los compañeros no se tomaban la molestia de abrirlo y hacer una evaluación crítica. Ante esta situación, hubo que comunicarles por correo electrónico que debían enviar cada término al foro para que los demás pudieran visualizarlo rápidamente, analizarlo y evaluarlo.

En relación al objetivo de potenciar el trabajo en grupo, al no haberse establecido mecanismos de control adecuados, fue imposible hacer un seguimiento de la participación individual de cada uno de los componentes del grupo en la elaboración de los términos del glosario. Por este motivo no se puede afirmar que trabajaran realmente en grupo, sino que más bien parece que se repartieron los conceptos entre los miembros de cada equipo, sin puesta en común antes de subirlos al foro. Por otro lado, y aunque pueda resultar paradójico, no fue posible evaluar de manera individualizada el trabajo realizado por cada alumno, ya que los términos figuraban como trabajo del equipo, de manera que finalmente se adjudicó a todos los miembros del grupo la misma nota.

Hay que destacar el interés de los estudiantes en utilizar el glosario como recurso didáctico para estudiar el examen final de la asignatura. Por ello, pidieron a las profesoras que estuviera completado al menos tres semanas antes de la fecha de examen. Conocer que el examen final consistiría en responder a cinco preguntas y que una o dos de ellas podían contestarse con este instrumento se convirtió, desde el primer momento, en una gran fuente de motivación. Los alumnos reconocieron que para el estudio de las primeras lecciones del temario, en las que se desarrollan conceptos generales de introducción al Derecho totalmente nuevos para ellos, el glosario constituía un buen recurso didáctico y les evitaba tener que recurrir a distintos manuales de Derecho cuyo vocabulario tampoco entendían.

Al final se pasó una encuesta sobre esta actividad. A continuación se reproducen las tablas con los resultados obtenidos en el cuestionario.

Los valores numéricos del cuestionario significan:

$1 \rightarrow$ Nada; $2 \rightarrow$ Poco; $3 \rightarrow$ Suficiente; $4 \rightarrow$ Bastante; $5 \rightarrow$ Mucho. 
a) Me ha gustado hacer este trabajo

$20 \% \rightarrow$ 5, Mucho; $20 \% \rightarrow$ 4, Bastante; $40 \% \rightarrow$ 3, Suficiente; $20 \% \rightarrow$ 2, Poco.

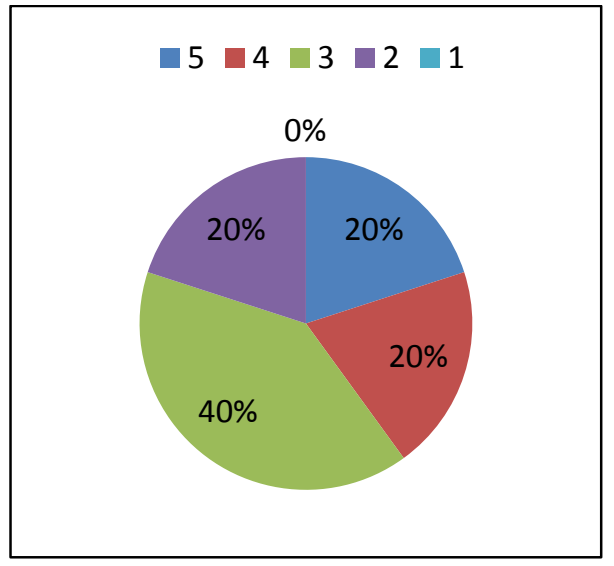

Fig. 1

b) He aprendido algo con este trabajo

$70 \% \rightarrow 4$, Bastante; $30 \% \rightarrow$ 3, Suficiente.

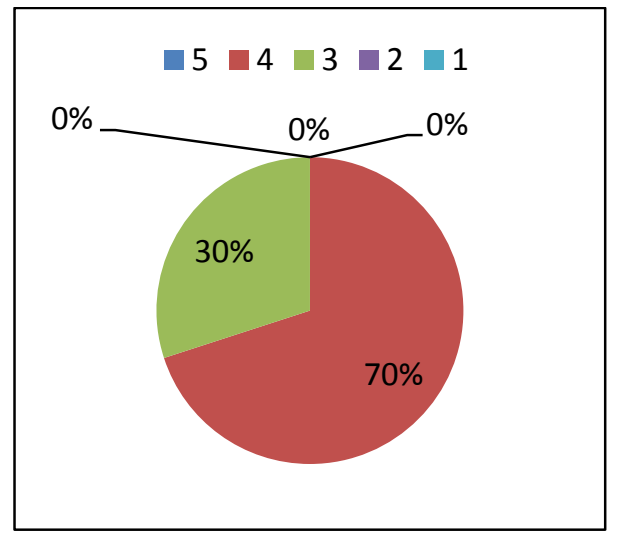

Fig. 2

\section{(c) EY-NC-ND 2015, Universitat Politècnica de València}

Congreso In-Red (2015) 
Elaboración de un glosario de términos jurídicos en la asignatura Fundamentos jurídicos y sociales para la ordenación territorial y medioambiental, grado de Geografía y Ordenación del Territorio

c) Considero que la realización de este trabajo me ha servido para estudiar la asignatura

$30 \% \rightarrow$ 5, Mucho; 30\% $\rightarrow$ 4, Bastante; $40 \% \rightarrow$ 3, Suficiente.

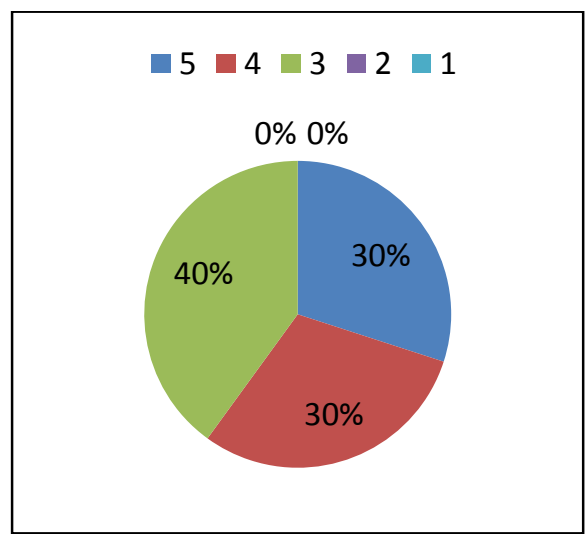

Fig. 3

d) Creo que este tipo de colaboración entre alumnos y profesores para la elaboración de materiales didácticos es muy útil y debería realizarse más a menudo

$50 \% \rightarrow 5$, Mucho; $30 \% \rightarrow$ 4, Bastante, $10 \% \rightarrow$ 3, Suficiente, $10 \% \rightarrow$ 2, Poco. 


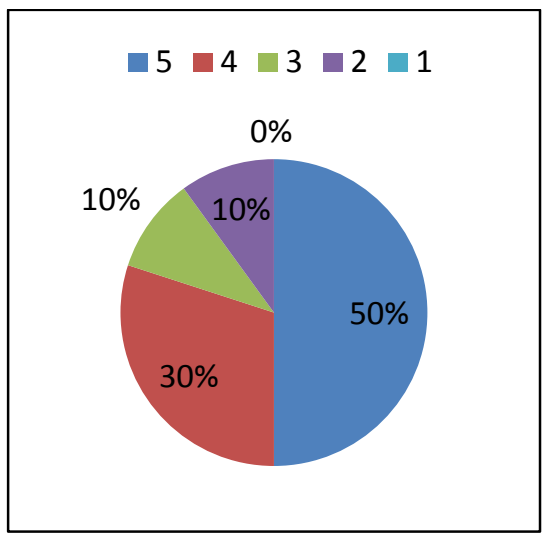

Fig. 4

De los resultados obtenidos en esta breve encuesta se colige que con el glosario de términos jurídicos se consiguió un recurso didáctico muy útil para el estudio de una asignatura jurídica introductoria que utiliza un lenguaje específico con términos completamente nuevos en el grado en Geografía y Ordenación del Territorio cuyo contenido se encuentra disperso en distintos manuales de Derecho Constitucional y Derecho Administrativo, de redacción compleja para alumnos de titulaciones no jurídicas. Por otra parte, al mismo tiempo que hacían el glosario, aprendían e iban tomando contacto con las lecciones del programa. De esta manera también se logró un aspecto esencial para mejorar el proceso de enseñanza-aprendizaje: que sea continuo y se desarrolle a lo largo de todo el semestre, en vez de realizar exclusivamente el estudio la semana previa al examen final. Ver figuras 2, 3 y 4 .

Sin embargo, como observamos en la figura 1, la realización de la actividad no les entusiasmó debido a la elevada carga de trabajos a la que tienen que atender en la evaluación continua de todas las asignaturas del grado, exposiciones orales, entrega de prácticas, etc.

También se mostraron muy reacios a evaluar los términos que sus compañeros enviaban al foro y pidieron a las profesoras que fueran ellas quienes evaluaran y decidieran aquéllos que estaban mejor definidos.

\section{Conclusiones}

Esta experiencia nos ha demostrado que el glosario de términos jurídicos resulta un instrumento práctico tanto para el profesor como para los alumnos, si bien es necesario definir bien la actividad y, sobre todo, establecer fórmulas para lograr una evaluación más adecuada.

Hemos comprobado que si este tipo de actividades no está alineada con la evaluación, los alumnos pierden interés porque, aunque saben que es un recurso didáctico interesante para el examen final, el esfuerzo que invierten en hacer el trabajo no está recompensado adecuadamente.

\section{(c)) EY-NC-ND 2015, Universitat Politècnica de València}

Congreso In-Red (2015) 
Elaboración de un glosario de términos jurídicos en la asignatura Fundamentos jurídicos y sociales para la ordenación territorial y medioambiental, grado de Geografía y Ordenación del Territorio

El glosario de términos ha sido realizado por el alumnado y, una vez revisado, se publicó para utilidad de todo el grupo. Esto facilitó el aprendizaje de términos jurídicos propios de la asignatura.

Se han analizado los resultados de las calificaciones del actual curso, convocatoria de febrero y, en comparación con el mismo periodo del curso pasado, se puede concluir que la incorporación de esta actividad ha sido positiva para el alumnado y ha contribuido a mejorar su aprendizaje y los resultados académicos.

En los resultados de la encuesta de valoración de la actividad, los alumnos han corroborado que el glosario era un instrumento útil y han adquirido conocimientos durante su elaboración.

Naturalmente también se detectaron problemas como la inseguridad de los alumnos, la reserva a exponer su trabajo para que fuera examinado y juzgado por todos los compañeros y, sobre todo, la reticencia a corregir el trabajo de otros compañeros.

Por tanto, la conclusión que podemos extraer es que en este tipo de herramientas de aprendizaje colaborativo es muy positivo. Aunque también es necesario establecer, por parte del profesor, unas reglas rígidas, definidas previamente tanto para su elaboración como para su evaluación. La realización de esta experiencia durante los próximos cursos académicos permitirá tener un feed-back para corregir algunas de las disfuncionalidades que han aparecido durante este primer año.

\section{Referencias}

DE CASTRO CABRERA, MC., JUAREZ CLAVAÍN, E., PERIAÑEZ GÓMEZ, F. (2014). "Mejora en la evaluación de una asignatura con grupos numerosos a través de cuestionarios y glosario de terminos”, Proyecto de innovación docente de la Universidad de Cádiz. PI_14_038, mes de junio de 2014. <http// www.uca.es/recursos/doc/.../677456473_8102014124952.pdf> [Consulta: 4 de mayo de 2015]

ECHARRETA, C., PRADOS, F., POCH, J., SOLER, J. (2009). "La competencia "el trabajo colaborativo”. Una oportunidad para incorporar las TIC en la didáctica universitaria. Descripción de la experiencia con la plataforma ACGM" en Uoc papers. Revista sobre la sociedad del conocimiento. $<$ http://uocpapers.uoc.edu> [Consulta: 22 de abril de 2015]

FLORES, M.S., FERNÁNDEZ PAMPILLÓN, A, LÓPEZ, J.A., MATESANZ, M. (2009). “El Glosario e-derecho: un modelo empírico de la información jurídica para la transmisión y comprensión del Derecho de Propiedad Intelectual en los campus virtuales universitarios” en Revista Iberoamericana de Sistemas, Cibernética e Informática, Volumen 6, num.1, págs 1-6. http://www.iiisci.org/Journal/riSCI/ [Consulta: 14 de mayo de 2015]

HOLGADO SÁEZ, C. (2011). "Las nuevas tecnologías en los Estudios de Derecho en el marco del EEES: sugerencias didácticas de actividades colaborativas con entornos virtuales” en Revista Jurídica de Investigación e innovación Educativa, núm. 3. <http://www.eumed.net/rev/rejie> [Consulta: 14 de mayo de 2015] 
María del Mar Villagrasa Rozas y María del Carmen de Guerrero Manso

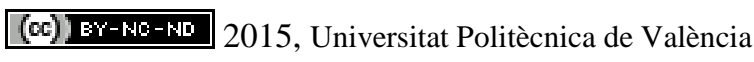

Congreso In-Red (2015) 